\title{
Storage ring lattice calibration using resonant spin depolarization
}

\author{
K. P. Wootton, ${ }^{1, *}$ M. J. Boland, ${ }^{1,2}$ W. J. Corbett, ${ }^{3}$ X. Huang, ${ }^{3}$ G. S. LeBlanc, ${ }^{2}$ M. Lundin, ${ }^{4}$ H. P. Panopoulos,,${ }^{1,}$ \\ J. A. Safranek, ${ }^{3}$ Y.-R. E. Tan, ${ }^{2}$ G. N. Taylor, ${ }^{1}$ K. Tian, ${ }^{3}$ and R.P. Rassool ${ }^{1}$ \\ ${ }^{1}$ School of Physics, The University of Melbourne, Melbourne, VIC, 3010, Australia \\ ${ }^{2}$ Australian Synchrotron, 800 Blackburn Road, Clayton, VIC, 3168, Australia \\ ${ }^{3}$ SLAC National Accelerator Laboratory, 2575 Sand Hill Road, Menlo Park, California 94025, USA \\ ${ }^{4}$ MAX IV Laboratory, Lund University, P.O. Box 118, SE-221 00 Lund, Sweden
}

(Received 22 February 2013; published 1 July 2013)

\begin{abstract}
This paper presents measurements of the GeV-scale electron beam energy for the storage rings at the synchrotron light source facilities Australian Synchrotron (AS) and SPEAR3 at SLAC. Resonant spin depolarization was employed in the beam energy measurement, since it is presently the highest precision technique and an uncertainty of order $10^{-6}$ was achieved at SPEAR3 and AS. Using the resonant depolarization technique, the beam energy was measured at various rf frequencies to measure the linear momentum compaction factor. This measured linear momentum compaction factor was used to evaluate models of the beam trajectory through combined-function bending magnets. The main bending magnets of both lattices are rectangular, horizontally defocusing gradient bending magnets. Four modeling approaches are compared for the beam trajectory through the bending magnet: a circular trajectory, linear and nonlinear hyperbolic cosine trajectories, and numerical evaluation of the trajectory through the measured magnetic field map. Within the uncertainty of the measurement the momentum compaction factor is shown to agree with the numerical model of the trajectory within the bending magnet, and disagree with the hyperbolic cosine approximation.
\end{abstract}

DOI: 10.1103/PhysRevSTAB.16.074001

PACS numbers: $29.27 . \mathrm{Hj}, 29.40 . \mathrm{Mc}, 41.75 . \mathrm{Ht}$

\section{INTRODUCTION}

This paper presents measurements of the momentum compaction factor using resonant spin depolarization, to calibrate the model of horizontal defocusing rectangular gradient bending magnets. We present experimental results in the storage rings of light sources SPEAR3 [1] and the Australian Synchrotron (AS) [2], which are modern light sources of intermediate energy with rectangular defocusing gradient dipoles in the double-bend achromat lattices [3]. In literature, there are very few measurements of the momentum compaction factor with rectangular gradient bending magnets. The momentum compaction factor was measured at ALS, with only a small departure from the model [4], however no details of the lattice model used in that study are available. At the time of that measurement, the bending magnets of the ALS lattice were all rectangular defocusing gradient magnets.

Electron beam energy measurements using resonant spin depolarization have been performed at storage rings for calibrating many aspects of the machine [4-8], most notably the absolute beam energy, beam energy stability,

\footnotetext{
*k.wootton@student.unimelb.edu.au

${ }^{\dagger}$ Present address: Centre for P.E.T., Austin Hospital, 145 Studley Road, Heidelberg, VIC 3084, Australia.

Published by the American Physical Society under the terms of the Creative Commons Attribution 3.0 License. Further distribution of this work must maintain attribution to the author(s) and the published article's title, journal citation, and DOI.
}

and momentum compaction factor. The technique is used because it is the highest precision energy measurement presently available, and the typical measurement accuracy is of order $10^{-5}-10^{-6}$. This is one of very few methods for measurement of the momentum compaction factor, which can be calculated from measurements of the synchrotron frequency [9]. Pioneering work on polarization was performed on $\mathrm{e}^{+}-\mathrm{e}^{-}$collider rings such as ACO [10,11], VEPP-2M [12,13], SPEAR [14], and LEP [15]. Our method of polarizing and depolarizing the beam follows the technique used at BESSY I [5], BESSY II [6], ALS [4], SLS [7], and ANKA [8]. Independent recent measurements at Diamond [16] and SOLEIL [17,18] achieve the same high precision. However, of the above storage rings, the ALS alone employs rectangular gradient bending magnets.

Many existing [1,19-22], upgrading [23] and planned rings [24-28] incorporate defocusing gradients into the main bending magnets, as part of a strategy to reduce the horizontal equilibrium emittance. The trajectory through rectangular gradient magnets does not follow a circular arc as in the case of pure dipole magnets, and the modeling of gradient bending magnets is challenging. In this work, the electron trajectory through the gradient dipoles is modeled using trajectories that are circular, linear hyperbolic cosine, nonlinear hyperbolic cosine, or numerical integration of the measured magnetic field [21].

\section{POLARIZATION THEORY}

A familiar description of storage ring accelerators is that particles are deflected according to their electrical charge, 
mass, and energy using electric and magnetic fields. These fields are arranged such that beams perform stable, oscillatory motion over many thousands of turns, which can be measured to great precision as a frequency spectrum. Measurement and control of resonances at the revolution and rf frequencies, betatron and synchrotron tunes, informs the global properties of the linear lattice [29]. Here, we exploit another property of the electron-its spin-to inform and calibrate our model of the dipole lattice of storage rings.

A thorough review of theory and experiments with polarized beams of protons, electrons, and muons was undertaken by Mane [30]. We will revisit the main theories of radiative polarization of electron beams, adiabatic resonant spin depolarization, and Møller scattering crosssection polarimetry in the following sections.

\section{A. Radiative polarization}

A beam of electrons in a storage ring with an initial random distribution of spin orientations (unpolarized) develops polarization over time, by the Sokolov-Ternov effect [31]. Under the action of emission of spin-flip photons, the population of beam electron spins aligns parallel or antiparallel with the main guide field of the bending magnets. The population of spin-up and spin-down particles is biased by the asymmetry of transition probabilities of spin-flip radiation [31], and the polarization $P(t)$ of the beam develops by [30]

$$
P(t) \approx P_{0}\left(1-e^{-t / \tau_{\mathrm{ST}}}\right),
$$

where time is denoted by $t$. The polarization $P(t)$ approaches an equilibrium [6]

$$
P_{0}=\frac{8}{5 \sqrt{3}} \frac{\oint B_{\perp}^{3} d s}{\oint\left|B_{\perp}^{3}\right| d s} .
$$

Magnetic fields are considered in the directions perpendicular $\left(B_{\perp}\right)$ and parallel $\left(B_{\|}\right)$to the curvilinear trajectory of the beam, $s$. In a storage ring, both vertical and radial magnetic fields are denoted here by $B_{\perp}$. To accommodate reverse bends and wiggler insertion devices, we integrate both $B_{\perp}$ and its absolute value $\left|B_{\perp}\right|$ around the trajectory $s$. By inspection, $P_{0}$ approaches a maximum for a storage ring without reverse bends or wiggler insertion devices, and for a beam of electron species with gyromagnetic factor $g_{e} \approx 2$, the theoretical maximum of polarization is $P_{0}=0.9238$ in an isomagnetic, planar ring [31]. The characteristic Sokolov-Ternov polarization time $\tau_{\mathrm{ST}}$ is given by [31]

$$
\tau_{\mathrm{ST}}^{-1}=\frac{1}{4 \pi \epsilon_{0}} \frac{5 \sqrt{3}}{8} \frac{\hbar \gamma^{5} r_{e}}{m_{e}} \frac{1}{\rho^{3}},
$$

where $\rho$ is the local bending radius, $m_{e}, r_{e}$ the classical electron mass and radius, $\epsilon_{0}$ the permittivity of free space, $\hbar$ the reduced Planck's constant, and $\gamma$ is the Lorentz factor of this relativistic electron beam. If the bending radius $\rho(s)$ varies around the ring circumference, rather than use the average value $\rho$ we make the substitution of the third synchrotron radiation integral [30,32],

$$
\frac{1}{\rho^{3}} \rightarrow I_{3}=\frac{1}{2 \pi R} \oint \frac{1}{|\rho(s)|^{3}} d s,
$$

where $R$ denotes the mean ring radius.

\section{B. Resonant spin depolarization}

Spin transport is described by the Thomas-BargmannMichel-Telegdi equation [33]. The electron spin precesses about the polarization axis at the spin precession frequency [15]

$$
\begin{aligned}
\vec{\Omega}_{\mathrm{BMT}}= & -\frac{q_{e}}{\gamma m_{e}}\left[\left(1+a_{e} \gamma\right) B_{\perp}+\left(1+a_{e}\right) B_{\|}\right. \\
& \left.-\left(a_{e} \gamma+\frac{\gamma}{1+\gamma}\right) \frac{\vec{\beta} \times \vec{E}}{c}\right],
\end{aligned}
$$

where $q_{e}$ is the electric charge and $a_{e}=\left(g_{e}-2\right) / 2$ the anomalous magnetic moment of the electron, and $c$ the speed of light in vacuum. As defined for Eq. (2), the direction of electric fields $\vec{E}$ are considered with respect to the relativistic velocity of the electron $\vec{\beta}=\vec{v} / c$. Normally the storage rings of light sources do not include any significant solenoid magnetic fields nor transverse electric fields, that is $B_{\|}=0, \vec{\beta} \times \vec{E}=0$. Hence, we can make the simplifying assumption that the electron spin precesses about a polarization axis which is antiparallel to $B_{\perp}$ (for bending magnets of a ring, a vertical magnetic field), at a frequency given by Eq. (5) which can be simplified to the spin tune $\nu_{\text {spin }}[15]$ :

$$
\nu_{\text {spin }}=a_{e} \gamma \equiv\left(\frac{g_{e}-2}{2}\right) \frac{E}{m_{e} c^{2}},
$$

where $E$ is the beam energy. If the beam is excited by a radial magnetic field $f_{\text {kick }}$ resonant at any harmonic to the spin tune, the polarization axis of the beam can be coherently rotated away from its equilibrium vertical orientation. The beam is hence resonantly depolarized at the frequency $f_{\text {kick }}=f_{\text {dep }}$.

\section{Current fundamental and experimental uncertainties}

The gyromagnetic factor $g_{e}$ for electrons has been measured to precision within the 12th significant figure [34]. We use the NIST CODATA values [35] for $a_{e}=$ $0.00115965218076(27)$ and $m_{e}=0.510998928(11) \mathrm{MeV}$. As a point of interest, with a relative uncertainty of $\Delta a_{e} / a_{e}=2.3 \times 10^{-10}, \Delta m_{e} / m_{e}=2.2 \times 10^{-8}$, the uncertainty in the electron mass has improved by almost an order of magnitude since 1994 [15], and hence the theoretical fundamental limit of uncertainty in the resonant 
TABLE I. Relative experimental uncertainties for AS and SPEAR3 experiments.

\begin{tabular}{lc}
\hline \hline Parameter & Relative uncertainty \\
\hline$a_{e}$ & $2.3 \times 10^{-10}$ \\
$m_{e}$ & $2.2 \times 10^{-8}$ \\
$f_{\text {rf }}$ & $1 \times 10^{-10}$ \\
$f_{\text {kick }}$ & $1 \times 10^{-7}$ \\
$f_{\text {dep }}$ & $1 \times 10^{-6}$ \\
\hline \hline
\end{tabular}

depolarization technique is reduced to approximately $\Delta E / E=2.2 \times 10^{-8}$. In practice, this limit remains orders of magnitude lower than other experimental uncertainties, as detailed for these experiments in Table I.

As outlined in Table I, the rf frequency $f_{\text {rf }}$ is calibrated to high precision and the excitation frequency $f_{\text {kick }}$ can be calibrated against a reference clock. Hence from Eq. (6), measurement of the spin tune $\nu_{\text {spin }}$ gives a direct measurement of the beam energy, with experimental uncertainty dominated by uncertainty in fitting the depolarizing frequency $f_{\text {dep }}$.

\section{Depolarizing effects}

The effective polarization time $\tau_{\text {eff }}$ is given by $[17,36]$

$$
\frac{1}{\tau_{\mathrm{eff}}}=\frac{1}{\tau_{\mathrm{ST}}}+\frac{1}{\tau_{\mathrm{dep}}}
$$

where $\tau_{\mathrm{ST}}$ represents the Sokolov-Ternov polarization time [Eq. (3)], and $\tau_{\text {dep }}$ a depolarization time governed by radial magnetic field errors [17]. Storage rings of several GeV achieve Sokolov-Ternov polarization times on the order of 15-20 minutes, with depolarization times exceeding several hours [37]. Because the depolarization effects have a much longer characteristic time, the effective polarization time is dominated by the Sokolov-Ternov polarization time.

A strong depolarizing resonance to be avoided is the choice of stored beam energy corresponding to integer spin tune [Eq. (6)] [11]. Also depolarizing is the overlap of the spin tune with betatron or synchrotron tunes. The vertical betatron tune has been usefully employed for resonant spin depolarization [14]. In that experiment, the vertical betatron tune was swept as the depolarizer. The width of the vertical betatron tune resonance limited the uncertainty in the beam energy measurement to approximately $\Delta E / E=10^{-4}$. In this experiment at the AS and SPEAR3, a feedback kicker is excited with a sinusoidal oscillation, because it can have a narrower frequency spread than the betatron tune.

\section{E. Møller scattering polarimetry}

Møller scattering is electron-electron scattering and occurs within a bunch in the storage ring. The polarimetry observable is the Møller scattering cross section of
Touschek scattered electrons [38-40]: the intrabunch cross section resulting from betatron oscillations. The particle loss rate $d N / d t$ is described in terms of the polarization $P(t)$ by $[12,30,37]$

$$
\frac{d N}{d t}=-\frac{N(t)^{2} c}{\sqrt{2} \gamma^{2} \sigma_{x} \sigma_{x^{\prime}} \sigma_{y} \sigma_{y^{\prime}} \sigma_{z}}\left[f_{1}+f_{2} P(t)^{2}\right]
$$

where the number of electrons per bunch is $N(t)$. For a stored beam of current $I(t)$ with equal current in several bunches, the bunch population $N(t) \propto I(t)$. The horizontal, vertical, and longitudinal beam dimensions are denoted by $\sigma_{x}, \sigma_{y}, \sigma_{z}$, and divergences denoted $\sigma_{x^{\prime}}, \sigma_{y^{\prime}}$ in the horizontal and vertical directions, respectively. The functions $f_{1}$ and $f_{2}$ can be treated for a given measurement as constants. Importantly, because $N(t) \propto I(t)$, an instantaneous normalized loss rate $R_{\text {norm }}$ can be defined as

$$
R_{\text {norm }}=\frac{1}{I(t)^{2}} \frac{d N}{d t} \propto f_{1}+f_{2} P(t)^{2}
$$

The normalized loss rate is the figure of merit used to evaluate changes in the level of beam polarization.

\section{DEPOLARIZATION EXPERIMENTS}

\section{A. Lattice and beam parameters}

Depolarization experiments were conducted at both the AS and SPEAR3 storage rings [41,42]. The pertinent design parameters of the two rings are summarized in Table II.

Using Eq. (6), the spin tune of a $3 \mathrm{GeV}$ electron beam was calculated as $\nu_{\text {spin }}=6.8081$, as presented in Table II. As outlined in Sec. II D, betatron tunes are depolarizing if overlapping with the spin tune. For the initial measurement of both rings, quadrupole strengths were changed to reduce the fractional vertical tune to approximately 0.1 .

\section{B. Polarization time}

For both the AS and SPEAR3 storage rings, an unpolarized beam of electrons was injected into the storage ring, which was observed to polarize over time. The measured

TABLE II. Storage ring design parameters.

\begin{tabular}{lcccc}
\hline \hline Parameter & & AS & SPEAR3 & \\
\hline Beam energy & $E$ & 3.00 & 3.00 & $\mathrm{GeV}$ \\
Lattice periodicity & $\ldots$ & 14 & 18 & $\ldots$ \\
Lorentz factor & $\gamma$ & 5871 & 5871 & $\ldots$ \\
Spin tune & $\nu_{\text {spin }}$ & 6.8081 & 6.8081 & $\ldots$ \\
Betatron tunes & $\nu_{x}$ & 13.290 & 14.130 & $\ldots$ \\
& $\nu_{y}$ & 5.216 & 6.194 & $\ldots$ \\
Bending radius & $\rho$ & 7.69 & 7.86 & $\mathrm{~m}$ \\
Circumference & $\mathrm{C}$ & 216.000 & 234.144 & $\mathrm{~m}$ \\
rf frequency & $f_{\mathrm{rf}}$ & 499.671 & 476.300 & $\mathrm{MHz}$ \\
Polarization time & $\tau_{\mathrm{ST}}$ & 807 & 1003 & $\mathrm{~s}$ \\
\hline \hline
\end{tabular}




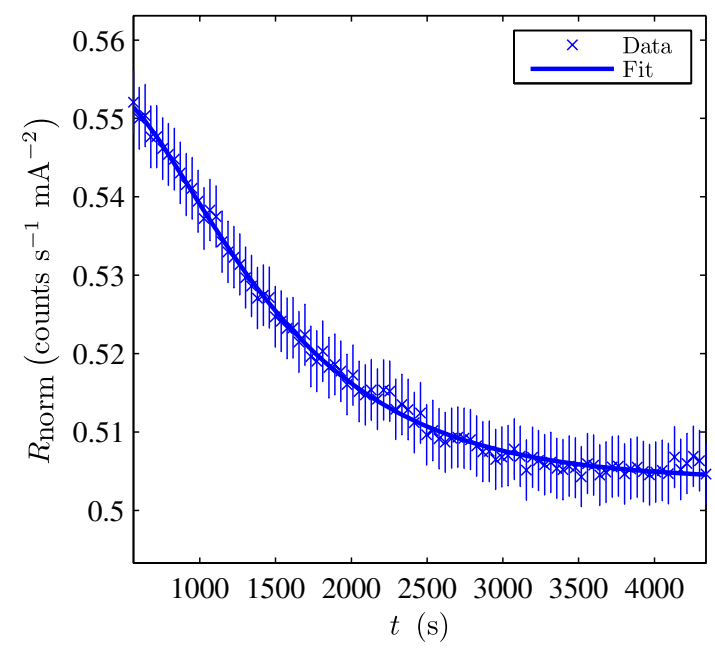

FIG. 1. Measurement of polarization time (AS). Fit to normalized count rate gives $\tau_{\text {eff }}=806 \pm 21 \mathrm{~s}$.

normalized loss rate $R_{\text {norm }}$ was fitted by Eqs. (1) and (9) to determine the polarization time. This is shown for the AS in Fig. 1, and for SPEAR3 in Fig. 2.

Using Eq. (3) and design parameters in Table II the Sokolov-Ternov polarization time was calculated. The measured $\tau_{\text {eff }}$ and theoretical $\tau_{\mathrm{ST}}$ are compared in Table III.

Importantly, for both rings a change in $R_{\text {norm }}$ is observed, which can be sensibly attributed to a change in polarization $P(t)$ by the Sokolov-Ternov effect. Hence, with this apparatus we will be able to observe the depolarization of the beam at the spin tune. Additional detail about the detector apparatus is given in Appendix A.

\section{Spin tune and beam energy measurement}

With the stored electron beam polarized, a time-varying radial magnetic field was applied to the beam through a fast

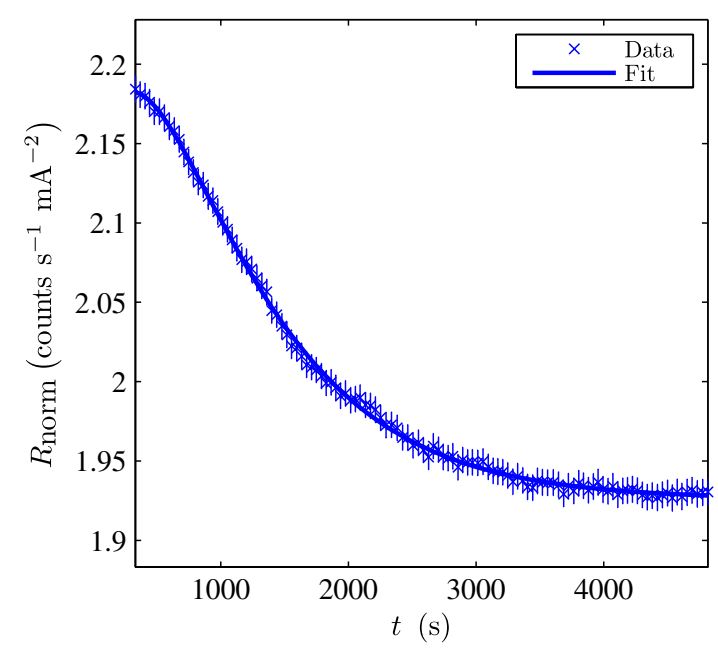

FIG. 2. Measurement of polarization time (SPEAR3). Fit to normalized count rate gives $\tau_{\text {eff }}=840 \pm 12 \mathrm{~s}$.
TABLE III. Polarization time in AS and SPEAR3.

\begin{tabular}{lcr}
\hline \hline Machine & Measured $\tau_{\text {eff }}(\mathrm{s})$ & $\tau_{\mathrm{ST}}(\mathrm{s})$ \\
\hline AS & $806 \pm 21$ & 807 \\
SPEAR3 & $840 \pm 17$ & 1003 \\
\hline \hline
\end{tabular}

kicker magnet and the oscillating frequency was scanned close to the predicted spin tune. Additional detail concerning the depolarizing kicker is given in Appendix B. As the magnetic field frequency crosses the spin tune the normalized loss rate increases indicating the beam is depolarized. Figure 3 shows a depolarizing event at AS where the change in the rate is clearly observable over and above the fluctuations due to counting statistics.

The uncertainty in the central frequency of the spin tune is not the same as the width of the spin tune resonance. The width of the spin tune resonance $\sigma_{f} / f_{\text {dep }} \approx 5 \times 10^{-6}$ was observed to be much larger than the uncertainty in the central frequency of the spin tune resonance $\Delta f_{\text {dep }} / f_{\text {dep }} \approx$ $2 \times 10^{-6}$. In addition, the width of the spin tune resonance is much narrower than the energy spread of the beam, which for this lattice is $\sigma_{E} / E \approx 1 \times 10^{-3}$ [2].

The beam energy for AS and SPEAR3 were extracted from spin tune frequency measurements, and the results are presented in Table IV. The high precision which was achieved with this energy measurement technique is exploited to measure the momentum compaction factor of the storage rings.

\section{Momentum compaction factor}

The momentum compaction factor is the change in circumference with change in energy $[29,43]$. For a set of rf frequencies, the beam energy was measured using the

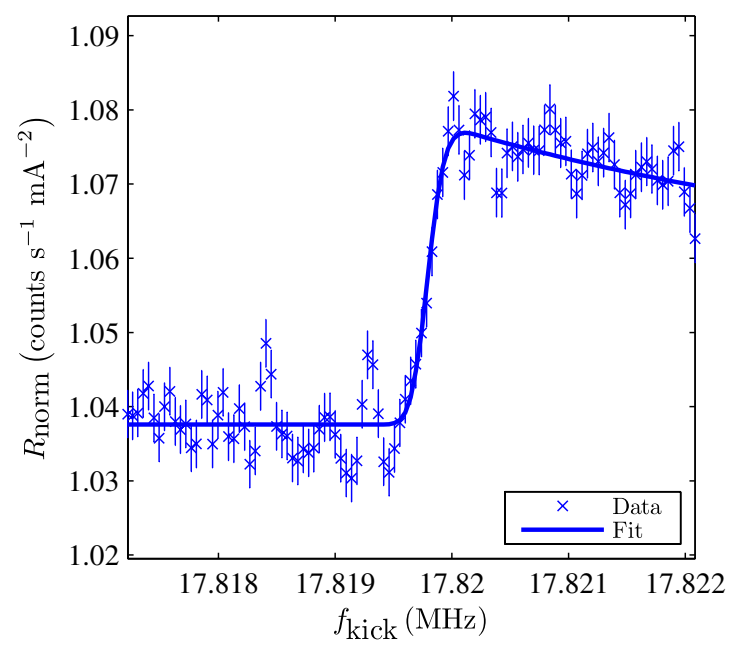

FIG. 3. Resonant spin depolarization (AS). A central frequency of $f_{\text {dep }}=17819790 \pm 30 \mathrm{~Hz}$ is fitted with a width of $\sigma_{f}=110 \pm 40 \mathrm{~Hz}$. An error function is fitted, allowing for the subsequent repolarization of the beam. 
TABLE IV. Measured spin tune and beam energy.

\begin{tabular}{lccc}
\hline \hline Machine & $f_{\text {dep }}(\mathrm{Hz})$ & $\nu_{\text {spin }}=a_{e} \gamma$ & $E(\mathrm{eV})$ \\
\hline AS & $17819790 \pm 30$ & $6.83859 \pm 0.00002$ & $3013416 \pm 9$ \\
SPEAR3 & $253620 \pm 20$ & $6.80192 \pm 0.00002$ & $2997251 \pm 7$ \\
\hline \hline
\end{tabular}

resonant spin depolarization technique. The rf frequency provides an accurate constraint on the circumference of the closed orbit, and resonant spin depolarization an accurate measurement of stored beam energy. In order to keep the beam energy stable, rf frequency feedback and fast-orbit feedback were turned off. The rf frequency was varied by small changes of 500 and $1000 \mathrm{~Hz}$ from the nominal frequency given in Table II, resulting in small changes to the stored beam energy. The corresponding change in beam energy was measured, as illustrated in Fig. 4 for AS. A similar measurement was implemented for SPEAR3. The measured momentum compaction factors of each ring are summarized in Table V. These values are later compared with calculated momentum compaction factor for the different models of the gradient dipoles in the rings as described in Sec. IV F.

\section{GRADIENT BENDING MAGNET MODELING}

As demonstrated in Sec. III D, we can exploit resonant depolarization to measure the momentum compaction factor of a storage ring. The momentum compaction factor $\alpha_{c}$ of a lattice can be calculated by integrating around the curvilinear trajectory $s$ as [44]

$$
\alpha_{c}=\frac{1}{C} \oint_{0}^{C} \frac{\eta_{x}(s)}{\rho(s)} d s
$$

where $C$ is the circumference, $\eta_{x}(s)$ the horizontal dispersion, and $\rho(s)$ the local bending radius. In previous work, several analytical models as well as a numerical model

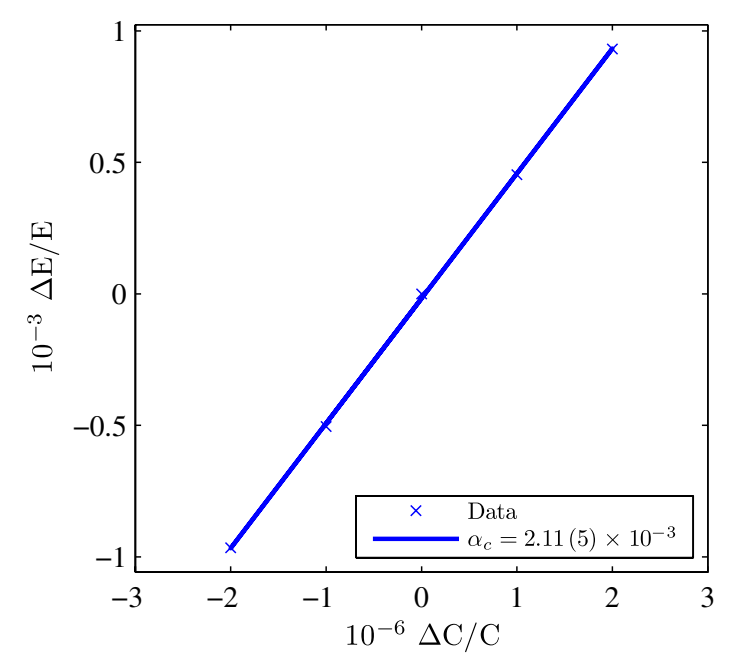

FIG. 4. Momentum compaction factor measurement (AS).
TABLE V. Momentum compaction factor measured using resonant spin depolarization.

\begin{tabular}{lc}
\hline \hline Machine & $\alpha_{c}$ \\
\hline AS & $0.00211 \pm 0.00005$ \\
SPEAR3 & $0.00164 \pm 0.00001$ \\
\hline \hline
\end{tabular}

were used to describe the trajectory of an electron beam through a rectangular gradient bending magnet [21], as used in the present experiments. Without a rigorous derivation, we quote the results of trajectories described by circular, analytical linear, analytical nonlinear, and numerical models. The model lattice optical functions and momentum compaction factor are analyzed and compared to measured values.

\section{A. Coordinates and parameters}

In this section of the paper, the coordinates of the beam trajectory will be given with reference to the straight, rectangular gradient bending magnet as illustrated in Fig. 5 for the AS storage ring.

This Cartesian coordinate system is right handed in $(x, y, z)$, with $x$ pointing radially outward from the storage ring center, $y$ vertically upwards, and $z$ parallel to the center line of the gradient magnet. The trajectory through a gradient bending magnet can be modeled as a beam traveling off center through a large quadrupole. We adopt a coordinate system [21], and define $(x, y, z)=(0,0, z)$ as the straight line along the center of this quadrupole, with $z=0$ at the longitudinal center of the quadrupole as shown in Fig. 5. The bending magnet will be considered to be centered at $(x, y, z)=\left(-x_{\mathrm{Q}}, 0,0\right)$, where $x_{\mathrm{Q}}=B_{0} / B_{1}$ and

$$
B_{\mathrm{n}}(x, 0, z)=\frac{\partial^{n} B(x, z)}{\partial x^{n}},
$$

and $n$ is the order of the transverse derivative of the magnetic field. This is further illustrated in Fig. 6.

The numerically evaluated trajectory will be evaluated in terms of a magnetic field map measured in the $(x, 0, z)$ plane, but components of the numerically integrated magnetic field will subsequently be specified in a curvilinear coordinate system $(u, v, s)$. The curvilinear system is right handed with $s$ tangential to the trajectory of the beam, $u$ perpendicular and radially outwards, and $v$ perpendicular and vertically upwards. The curvilinear system is selected for the numerical trajectory because it is easy to implement curvilinear magnetic elements in existing accelerator tracking codes.

Parameters of the bending magnet are summarized in Table VI.

\section{B. Circular arc trajectory}

We make the simplifying assumption that the trajectory is approximated by a circular arc, as a base against which 


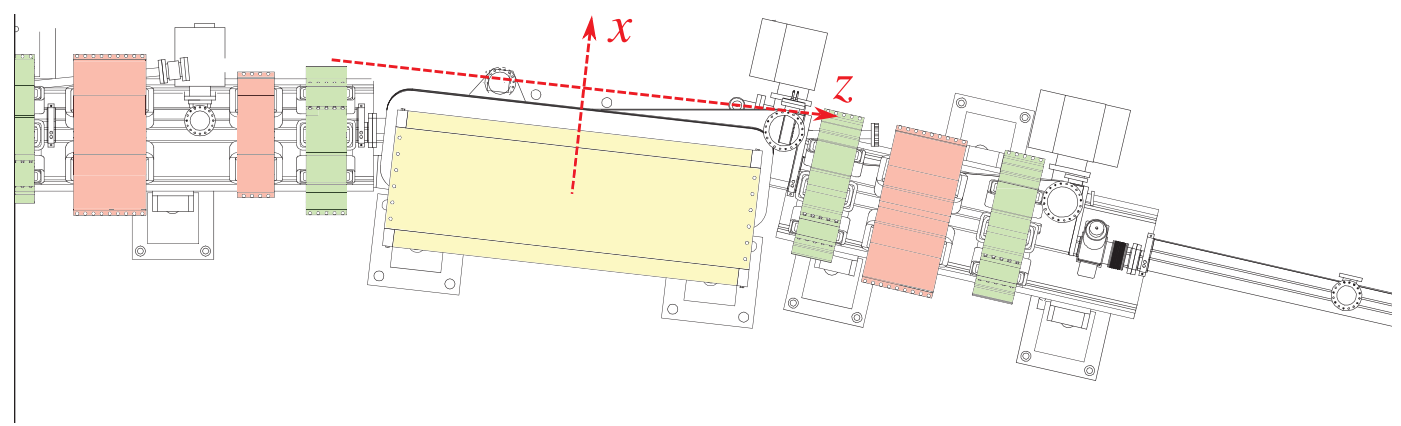

FIG. 5. Half sector of the AS storage ring [2]. The rectangular gradient bending magnet is shown in yellow, quadrupoles in red, and sextupoles in green.

to make comparisons. Ignoring the horizontal defocusing gradient, we approximate the pole profile as a pure dipole.

For the beam rigidity $B \rho$, bending angle $\theta$, and effective length $L_{\text {eff }}$ given in Table VI, the mean dipole field and bending radius are presented in Table VII. Unless otherwise specified only the vertical component of the magnetic field on the midplane will be used in the following analysis and discussion, i.e., $B(x, z)=B_{y}(x, 0, z)$.

In the coordinate system given, the circular trajectory $x_{\text {circ }}(z)$ within the magnet is expressed by

$$
x_{\text {circ }}(z)=\rho \sqrt{1-(z / \rho)^{2}}-x_{\mathrm{Q}} .
$$

Outside the effective length of the dipole $L_{\text {eff }}$, the trajectory is straight.

\section{Linear hyperbolic cosine trajectory}

The field profile of a gradient bending magnet can be considered to be the field of a quadrupole of very large bore, laterally offset from the center line of the bending magnet. This quadrupole is considered to have a strength, $k$, which is defined in terms of the defocusing gradient $B_{1}$ and beam rigidity $(B \rho)$ by $k=B_{1} /(B \rho)$. The equation of motion of the beam through this quadrupole is given by [21]

$$
x^{\prime \prime}(z)=\frac{B(x, z)}{B \rho}\left[1+x^{\prime}(z)^{2}\right]^{3 / 2},
$$

where the prime denotes the derivative along $z$. A linear solution to Eq. (13) can be obtained by making the

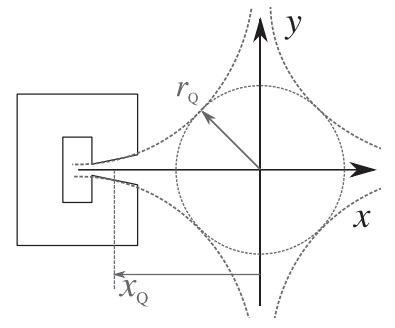

FIG. 6. Description of gradient bending magnet field by the field of a single quadrupole of radius $r_{\mathrm{Q}}$, laterally offset from the nominal center line of the bending magnet by a distance $x_{\mathrm{Q}}$ [21]. approximation $x^{\prime}(z)=0$. We quote the trajectory evaluated using this linear approximation, and direct the interested reader to its derivation [21]. The beam trajectory $x_{\mathrm{L}}(z)$ from magnet center to exit (and symmetrically, entrance) is expressed by

$$
\begin{gathered}
x_{\mathrm{L}}(z)=x_{\mathrm{L}}(0) \cosh (\sqrt{k} z), \\
x_{\mathrm{L}}(0)=\frac{\tan (\theta / 2)}{\sqrt{k} \sinh \left(\sqrt{k} L_{\text {eff }} / 2\right)} .
\end{gathered}
$$

The deflection of the beam is chosen to be symmetric longitudinally about the magnet center $z=0$ so that the magnet deflects the beam through a bending angle $\theta / 2$ within the length $L_{\text {eff }} / 2$, with maximum orbit amplitude at the magnet center.

\section{Nonlinear hyperbolic cosine trajectory}

A nonlinear analytic solution describing the horizontal trajectory of the electron through the bending magnet can be obtained on substitution of the linear solution given by Eq. (14) into the equation of motion, Eq. (13). This nonlinear analytic solution is given by [21]

TABLE VI. Storage ring bending magnet parameters.

\begin{tabular}{lcccc}
\hline \hline Parameter & Symbol & AS & SPEAR3 & units \\
\hline Beam rigidity & $B \rho$ & 10 & 10 & $\mathrm{~T} \mathrm{~m}$ \\
Bending angle & $\theta$ & $2 \pi / 28$ & $2 \pi / 34$ & $\mathrm{rad}$ \\
Defocusing gradient & $\partial B / \partial x$ & 3.35 & 3.63 & $\mathrm{~T} \mathrm{~m}^{-1}$ \\
Iron length & $L_{\text {iron }}$ & 1.700 & 1.450 & $\mathrm{~m}$ \\
Effective length & $L_{\text {eff }}$ & 1.726 & 1.505 & $\mathrm{~m}$ \\
\hline \hline
\end{tabular}

TABLE VII. Circular radius approximation.

\begin{tabular}{lcccr}
\hline \hline Parameter & Symbol & AS & SPEAR3 & \\
\hline Effective field & $B_{0}$ & 1.300 & 1.228 & $\mathrm{~T}$ \\
Bending radius & $\rho$ & 7.692 & 8.144 & $\mathrm{~m}$ \\
\hline \hline
\end{tabular}




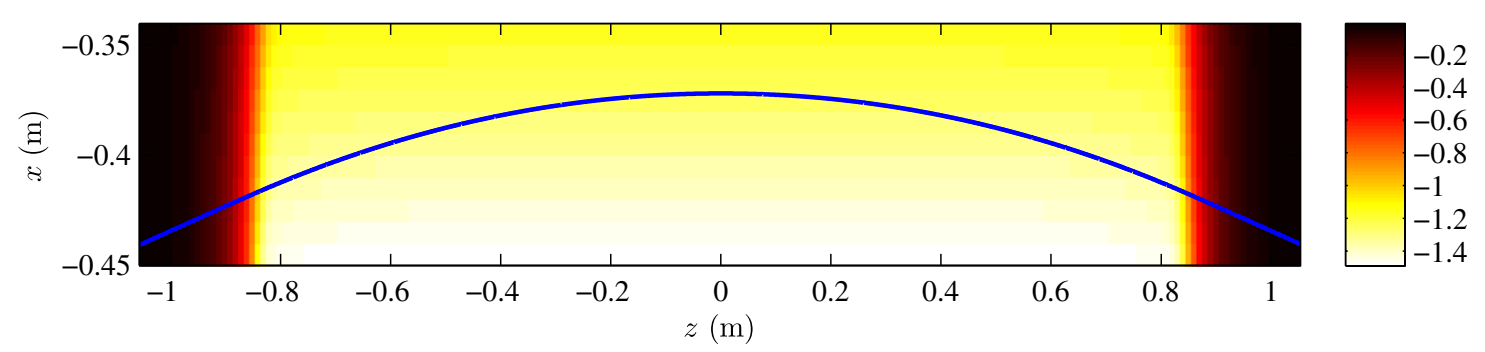

FIG. 7. Vertical component of magnetic field of AS horizontally defocusing gradient bending magnet, measured with a Hall probe. The $x-z$ axes corresponds to the coordinate system of Fig. 5. The numerically evaluated trajectory is represented by a solid blue line. The color scale shows the vertical component of the magnetic field $B_{y}(x, 0, z)$ in units of Tesla.

$$
\begin{aligned}
x_{\mathrm{NL}}(z)= & x_{\mathrm{NL}}(0)-\frac{1}{\sqrt{k}}\left[\sin ^{-1}\left(\frac{\cosh (\sqrt{k} z)}{\sqrt{1+1 /\left[k x_{\mathrm{NL}}(0)^{2}\right]}}\right)\right. \\
& \left.-\sin ^{-1}\left(\frac{1}{\sqrt{1+1 /\left[k x_{\mathrm{NL}}(0)^{2}\right]}}\right)\right]
\end{aligned}
$$

where

$$
x_{\mathrm{NL}}(0)=\frac{\tan (\theta / 2)}{\sqrt{k} \sinh \left(\sqrt{k} L_{\mathrm{eff}} / 2\right) \sqrt{1+\tan ^{2}(\theta / 2)}} .
$$

\section{E. Numerically integrated trajectory using measured magnetic field data}

A model of the AS bending magnet can be created by using a group of sector dipole elements with higher order multipole field components (quadrupole, sextupole, and octupole). This method has also been implemented for modeling the SPEAR3 ring [45], and has the benefit of ensuring that particle tracking is symplectic.

The magnetic field map of the defocusing gradient bending magnet was measured by a three-axis Hall probe on the horizontal midplane to give $B_{x, y, z}(x, 0, z)$. The vertical component of the magnetic field $B_{y}(x, 0, z)$ is presented in Fig. 7. Superimposed on the map is the trajectory of a $3 \mathrm{GeV}$ electron passing through the magnet as calculated using the numerical integration method.

The vertical magnetic fields experienced by the electron while traversing the gradient magnet can be approximated by calculating the coefficients of the Taylor expansion of the local magnetic field relative to the trajectory. As outlined in Sec. IVA, this curvilinear system is selected for the numerical trajectory because it is easy to implement a sequence of curvilinear magnetic elements in existing accelerator tracking codes.

The first step in the calculation is a coordinate transformation from the Cartesian $(x, y, z)$ coordinates to a curvilinear coordinate system $(u, y, s)$, where $s$ is tangential and $u$ perpendicular to the reference trajectory. Therefore, all field components are evaluated as

$$
B_{n}(u, s)=\frac{\partial^{n} B(u, s)}{\partial u^{n}} .
$$

The $n=0,1,2,3$ order coefficients are correspondingly referred to as the dipole, quadrupole, sextupole, and octupole components of the magnetic field. The field components for the AS bending magnet are shown in Fig. 8.

The horizontal trajectory of the particle through the measured magnetic field (blue curve in Fig. 7) is calculated by numerically solving Eq. (13) with a fourth order RungeKutta integrator (with no variable step size). The trajectory is constrained to longitudinal symmetry, i.e. $x\left(-L_{\text {eff }} / 2\right)=$ $x\left(L_{\text {eff }} / 2\right), x^{\prime}\left(-L_{\text {eff }} / 2\right)=x^{\prime}\left(L_{\text {eff }} / 2\right)$, and a deflection of $\int B_{0}(s) d s=(B \rho) \theta$.

The numerical trajectory is modeled with field components given by Eq. (18). The bending magnet model is constructed by performing piecewise integration of the field map along the trajectory $s$ at points $s_{i}$. For the $i$ th slice, the integrated multipole component is given by (a) Dipole

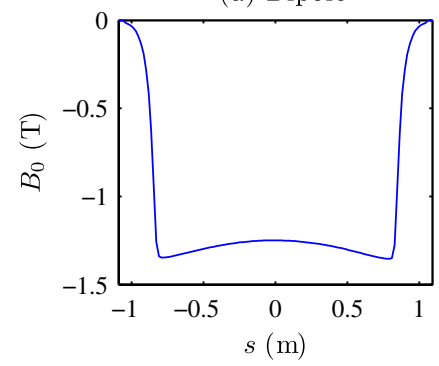

(c) Sextupole

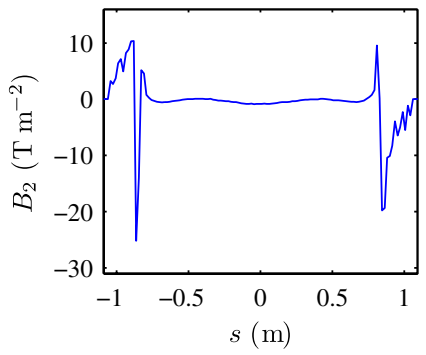

(b) Quadrupole

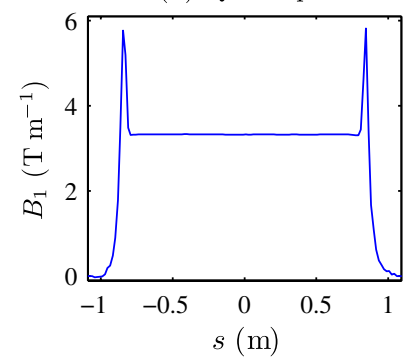

(d) Octupole

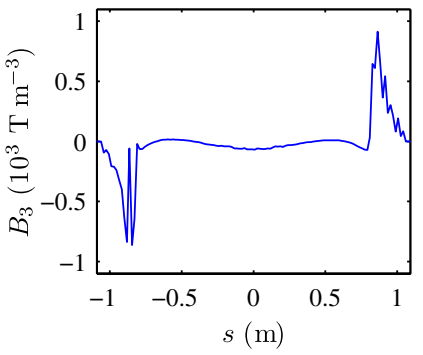

FIG. 8. Field components $B_{n}(u, s)$, along the curvilinear trajectory plotted in Fig. 7. (a) Dipole component. (b) Quadrupole component. The peaks at the ends of the bending magnet are due to edge focusing. (c) Sextupole component. (d) Octupole component. 


$$
m_{n}^{i}=\frac{1}{s_{i+1}-s_{i}} \int_{s_{i}}^{s_{i+1}} \frac{\partial^{n} B(0, s)}{\partial u^{n}} \partial s
$$

evaluated about the center of the trajectory $u=0$. This sequence of sector elements describing the bending magnet model can readily be incorporated in an accelerator lattice model. Because the edge effects are encapsulated by the modeling approach, the individual segments are modeled with higher order multipole components $m_{n}^{i}$.

\section{F. Evaluation of modeling approaches}

In the preceding subsections, different analytical approaches were presented for the modeling of a straight, rectangular gradient bending magnet. We propose to compare the measured and modeled momentum compaction factor as an evaluation of these models. As defined in Eq. (10), the momentum compaction factor depends upon

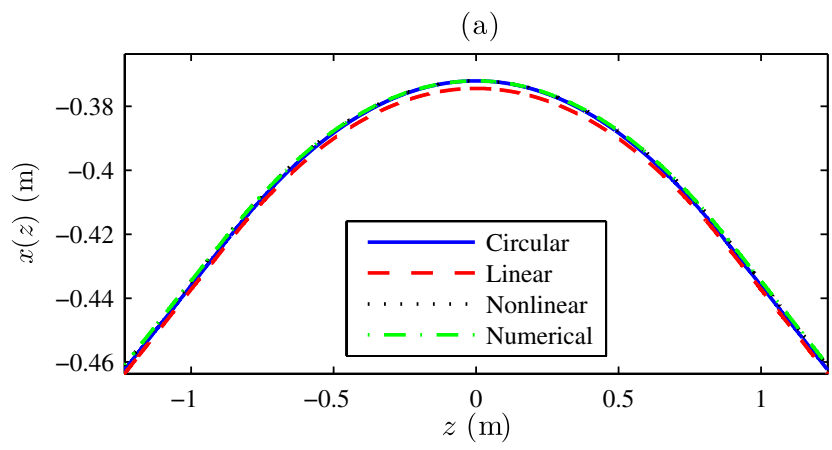

(b)

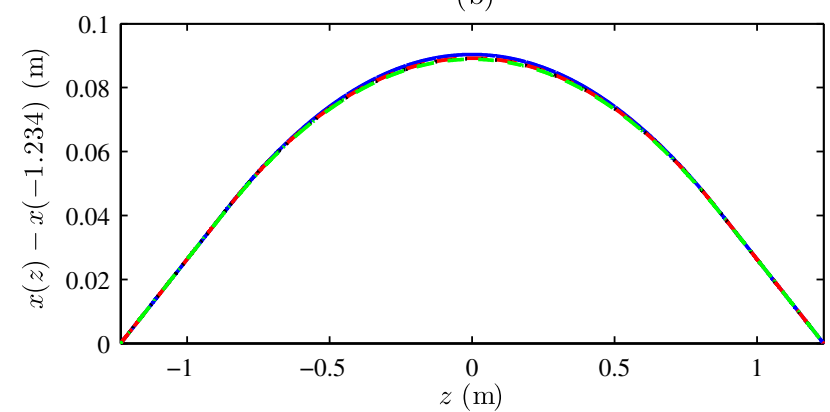

(c)

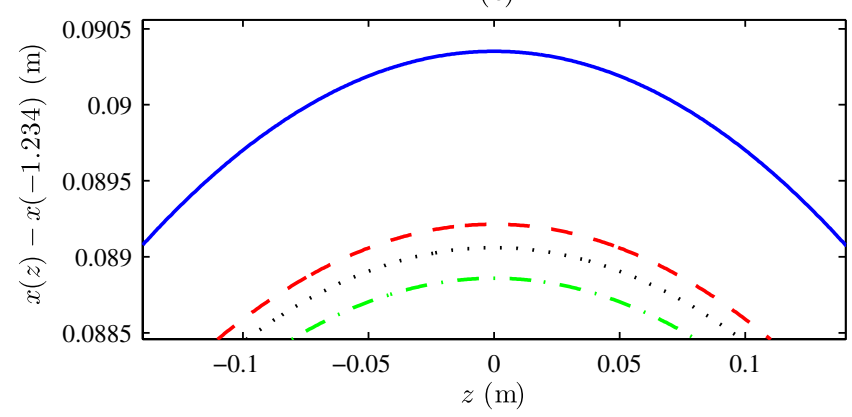

FIG. 9. (a) Electron beam trajectories in coordinates $(x, z)$ for each of the four models described in Sec. IV, with equal entrance and exit angles. (b) Translation in $x$ of trajectories in (a) by $x(z)-x(-1.234)$, to give equal entrance and exit positions and angles for each model. (c) Magnification of trajectories in (b). both the bending radius $\rho(s)$ and the horizontal dispersion $\eta_{x}(s)$. In this section, we will determine and evaluate both of these.

The trajectories of each of the four models are plotted in Fig. 9. As illustrated in Figs. 9(b) and 9(c), we compare the four trajectories relative to the initial position $x(z=-1.234)$. The circular trajectory approximation gives the greatest deviation from the numerically evaluated trajectory.

It is easier to see the local changes of the trajectory by inspecting the local bending radius of these four modeling approaches. The bending field $B_{0}(z)$ of the gradient bending magnet is described by a virtual quadrupole of transverse quadrupole gradient $B_{1}$, and hence the dipole field varies in the longitudinal coordinate $z$ by $B_{0}(z)=B_{1} x(z)$. As a result, the local bending radius $\rho(z)$ is given by

$$
\rho(z)=p /\left(q_{e} B_{0}\right)=p /\left[q_{e} B_{1} x(z)\right]
$$

where $p$ is the momentum of the beam electron. The local bending radii of each of the four models are plotted in Fig. 10. There is longitudinal variation in the bending radius $\rho(z)$, with the highest bending fields corresponding to lower bending radii at large $z$, as highlighted in Fig. 10(b). This results in a trajectory with greatest bending near the extrema of the bending magnet, and less bending in the center.

We can also compare the lattice parameters $[29,43]$ with bending magnets modeled by the linear hyperbolic and

(a)

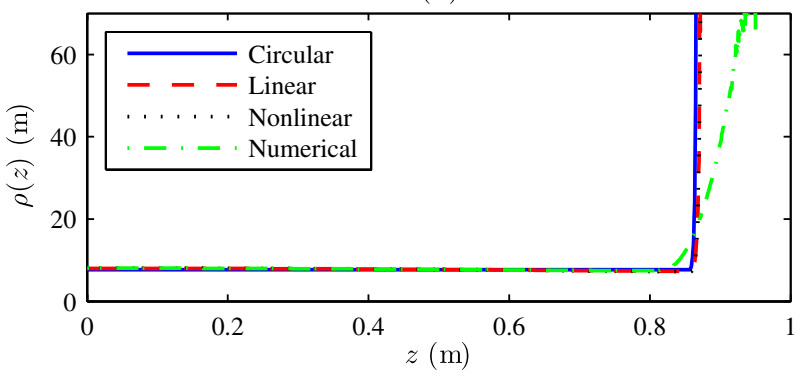

(b)

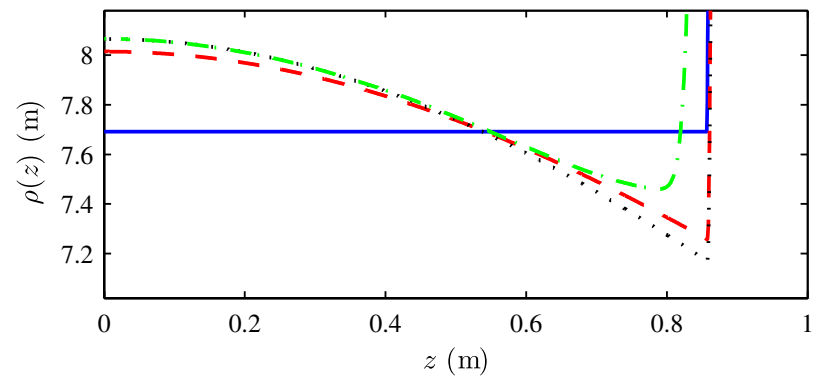

FIG. 10. Bending radius $\rho$ as a function of the longitudinal coordinate $z$, for each of the four models described in Sec. IV. (a) Bending radius $\rho(z)$ with longitudinal position $z$, highlighting the longitudinal extent of the fringe field in the numerical model. (b) Magnification of (a), highlighting variation of bending radius within the iron length of the magnet. 
numerical trajectories. The storage ring is simulated using the ACCELERATOR TOOLBOX (AT) code [46] for each of the analytical linear model given by Eq. (14), and numerically evaluated bending magnet fields given by Eq. (19). The equation for the path length of the trajectory using these models is given explicitly in Ref. [21]. Linear and numerical models of the trajectory are specifically compared, as the linear method is commonly implemented in accelerator tracking codes, and elements commonly included in accelerator tracking codes can be used to yield the numerically evaluated trajectory. For each model, the three families of storage ring quadrupoles are matched to give the same betatron tunes and horizontal dispersion in the straights. Figure 11 shows the difference between the betatron and dispersion functions for the AS lattice.

We observe in Fig. 11(b) that the solution of quadrupole strengths for matched tunes and dispersion in the center of the straights yields a significant difference in the dispersion and betatron functions. As an input to the momentum compaction factor, we focus on the change in the difference between the dispersion functions, which at the beam position monitor (BPM) in the center of the arc is a peak difference of $\eta_{x \mathrm{NUM}}-\eta_{x \mathrm{~L}}=4.8 \mathrm{~mm}$. The measured

(a)

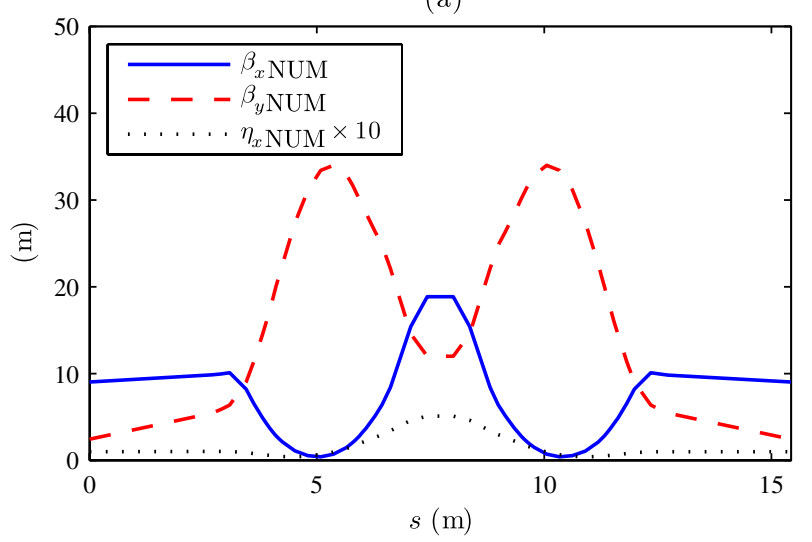

(b)

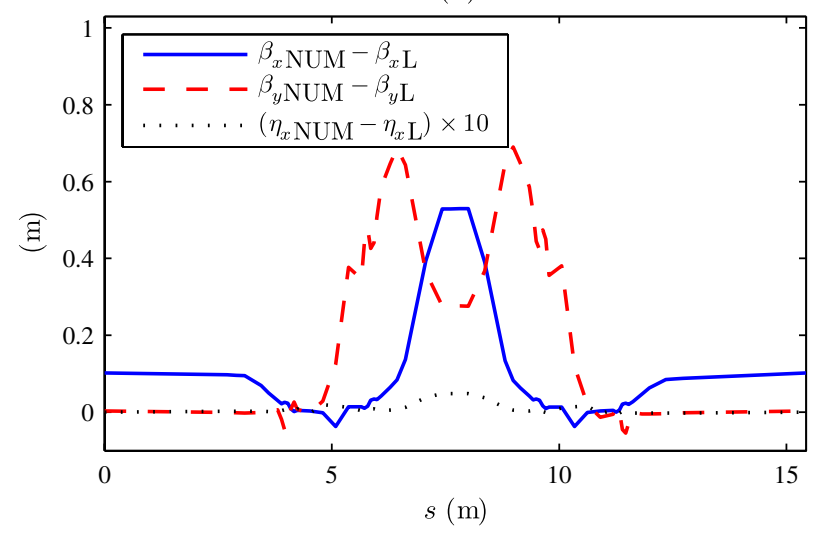

FIG. 11. (a) Lattice functions evaluated using numerical model for trajectory, fitting quadrupoles for the $0.1 \mathrm{~m}$ dispersion lattice of the AS [2]. (b) Difference between lattice functions of numerical and linear hyperbolic cosine trajectory. dispersion function $\eta_{x \text { MEAS }}$ is compared to numerical and linear models in Fig. 12.

For the horizontal dispersion shown in Fig. 12, the mean and standard deviation of the difference between the measured and model dispersion of the BPMs in the center of the arcs is for the linear model $\eta_{x \text { MEAS }}-\eta_{x \mathrm{~L}}=$ $5.8 \pm 1.4 \mathrm{~mm}$, and for the numerical model $\eta_{x \mathrm{MEAS}}-$ $\eta_{x \mathrm{NUM}}=-0.6 \pm 1.4 \mathrm{~mm}$. Hence, the measured dispersion function agrees with the numerical model to within the limits of this lattice calibration. The difference between the measured dispersion and linear model is attributable to the difference between the linear and numerical models.

As presented in Sec. III D, to evaluate which is a more accurate model of the bending magnet, we have measured the momentum compaction factor to high precision using resonant spin depolarization. Measured and modeled values of the momentum compaction factors of both lattices are compared in Table VIII.

Within the uncertainty of the measurement the momentum compaction factor is shown to agree with the numerical model of the trajectory within the bending magnet, and disagree with the linear hyperbolic cosine approximation. The accuracy of the numerical model comes from using the

(a)

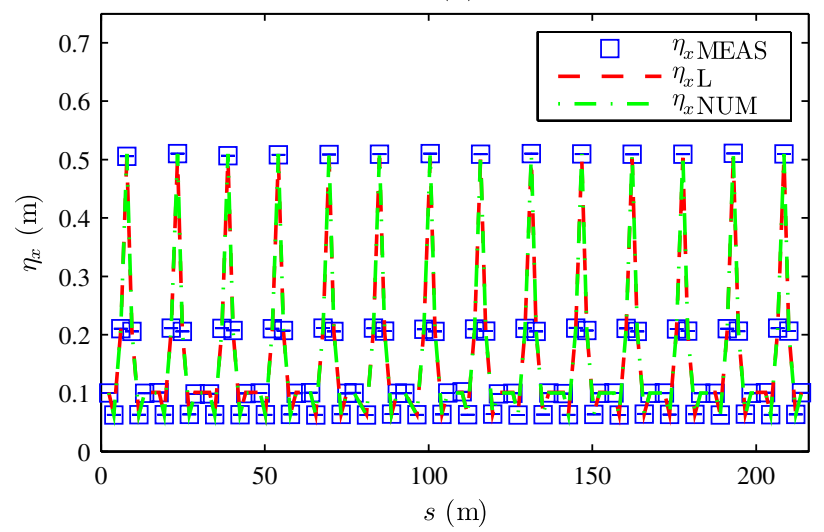

(b)

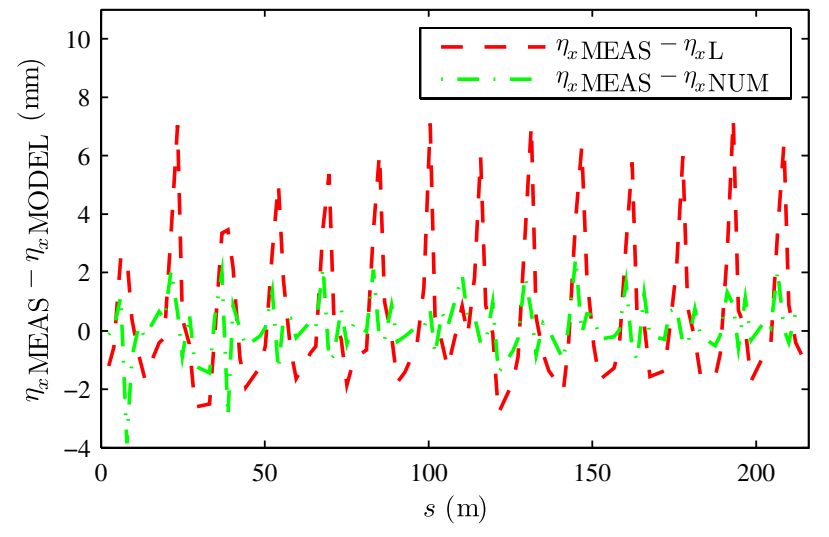

FIG. 12. (a) Measured and model dispersion functions for the AS. (b) Difference between measured dispersion, and dispersion evaluated using linear hyperbolic and numerical trajectory. 
TABLE VIII. Momentum compaction factor.

\begin{tabular}{lcc}
\hline \hline & AS & SPEAR3 \\
\hline Linear hyperbolic cosine & 0.00205 & 0.00162 \\
Numerical & 0.00211 & 0.00165 \\
Measured & $0.00211 \pm 0.00005$ & $0.00164 \pm 0.00001$ \\
\hline \hline
\end{tabular}

correct distribution of the dipole field component as illustrated in Figs. 8 and 10, subsequently giving a better model for the horizontal dispersion as illustrated in Fig. 12.

\section{DISCUSSION}

Resonant spin depolarization has been usefully employed previously at rings to confirm effects principally related to beam energy stability. In the present work, we benefit from the precision of the technique in the calibration of lattice models of gradient dipole magnets. We have compared the momentum compaction factor for analytic linear and numerically evaluated models, and measurements of storage ring lattices incorporating rectangular gradient bending magnets.

As a modeling technique, the numerical evaluation of trajectory could find potential application with various proposed accelerators. The design of present and future third-generation storage ring light sources already consider this approach $[22,47]$. Ultimate storage ring light sources [24-28] plan to employ gradient dipole magnets as part of a strategy to reduce the equilibrium horizontal emittance.

Fixed-field, alternating gradient accelerators are enjoying a recent resurgence in interest [48]. In particular, scaling lattices employing gradient dipoles $[49,50]$ could find benefits to trajectory and focusing from numerical modeling, as well as nonscaling lattices with real quadrupoles at large transverse offsets [51]. Also, with beams of antiproton species, this modeling approach could prove useful to the Recycler ring at Fermilab [52]. For proton accelerators, this numerical evaluation of trajectory has been demonstrated to natively account for modeling of magnet fringe fields [53].

\section{CONCLUSION}

The beam energy was measured for two electron storage rings AS and SPEAR 3 with defocusing gradient bending magnets. Resonant spin depolarization was employed, achieving an uncertainty of order $10^{-6}$ in the beam energy. To measure the momentum compaction factor, the rf frequency provided an accurate constraint on the circumference of the closed orbit, and resonant spin depolarization an accurate measurement of stored beam energy. Measurements and models of the momentum compaction factor were compared to evaluate analytical and numerical models of the beam trajectory through a defocusing gradient dipole magnet.

Armed with precision measurements, we made a critical evaluation of the suitability of different modeling ap- proaches of the electron beam trajectory. The trajectory through the gradient dipoles is modeled using circular, linear hyperbolic cosine, nonlinear hyperbolic cosine approximations, and numerical integration of the measured magnetic field. Within the uncertainty of the measurement the momentum compaction factor is shown to agree with the numerical model of the trajectory within the bending magnet, and disagree with the hyperbolic cosine approximation. Linear and numerical models of the trajectory are specifically compared, as the linear method is commonly implemented in accelerator tracking codes, and elements commonly included in accelerator tracking codes can be used to yield the numerically evaluated trajectory.

This is an extended article of measurements presented at recent International Particle Accelerator Conferences [41,42].

\section{ACKNOWLEDGMENTS}

Portions of this research were carried out at the Stanford Synchrotron Radiation Lightsource, a Directorate of SLAC National Accelerator Laboratory and an Office of Science User Facility operated for the U.S. Department of Energy Office of Science by Stanford University. Parts of this research were undertaken on the storage ring at the Australian Synchrotron, Victoria, Australia. K. P.W. thanks P. Kuske (BESSY), J. Zhang (LAL, Orsay), and I.P.S. Martin (Diamond) for useful discussions at and following IPAC'10 and IPAC'11.

\section{APPENDIX A: DETECTOR CHOICE}

The change in beam polarization is observed in the normalized loss rate $R_{\text {norm }}$, given by Eq. (9). Two main approaches are considered in literature: evaluation of the Touschek lifetime from the storage ring DC current

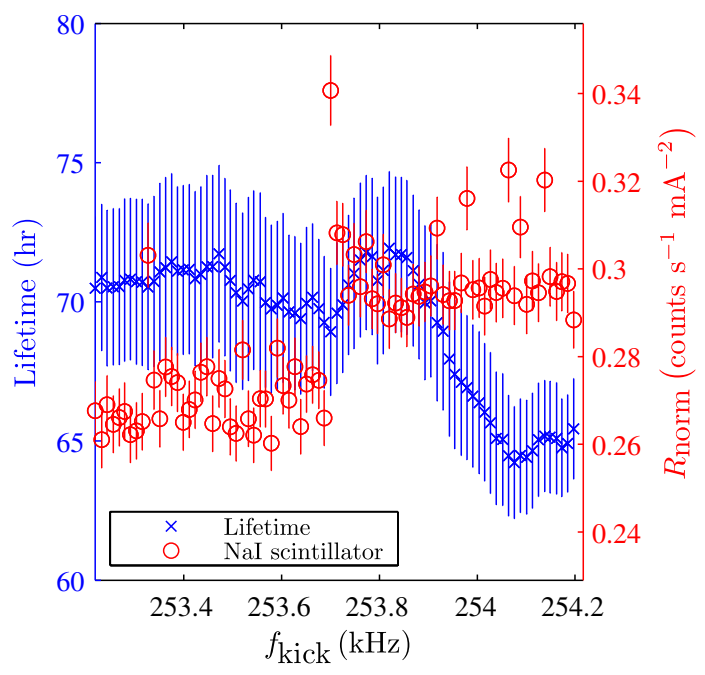

FIG. 13. Resonant depolarization measured for SPEAR3 using $\mathrm{NaI}$ scintillator and DCCT, increasing the excitation frequency in time. The lifetime is calculated from 30 samples of the DCCT. 
transformer (DCCT), and detection of the electromagnetic shower from Touschek scattered beam particles striking the vacuum chamber when lost [5,54]. Figure 13 presents measurements of a depolarization using each of these techniques.

Figure 13 shows that both approaches could work in the identification of a depolarization. The measured NaI loss monitor count rate responds to the resonant depolarization within $1 \mathrm{~s}$, while calculation of the lifetime with small uncertainty requires approximately $30 \mathrm{~s}$ of measurements of the stored beam current and the drop in lifetime is observed approximately $30 \mathrm{~s}$ after the depolarization. The beam lifetime is calculated from the time derivative of the stored beam current, while the loss monitor measures the absolute loss rate, which is the derivative of the beam current with respect to time.

For the technique of resonant spin depolarization it is best to have a beam lifetime dominated by Touschek scattering. One of the simplest ways to control this is to alter the fill pattern in the storage ring to maximize the single bunch current.

The beam loss monitor was a $75 \mathrm{~mm}$ diameter $\mathrm{NaI}$ scintillator and photomultiplier tube at AS, and $50 \mathrm{~mm}$ diameter at SPEAR3. For both experiments, the scintillator was installed in the orbit plane of the ring, on the inner side of the vacuum chamber. At SPEAR3, the detector was installed adjacent to the scraper defining the minimum energy aperture of the SPEAR3 storage ring, to maximize the count rate. This is immediately downstream of the central focusing quadrupole, which is the point of maximum horizontal dispersion in one of the double-bend achromat arc cells. At AS, the detector was installed at the upstream end of an insertion straight, where the horizontal dispersion is $0.1 \mathrm{~m}$.

In summary, the change in polarization needs to be quite large to observe the depolarization using the beam lifetime. From a beam physics perspective, large changes of polarization are not necessary to measure the beam energy: one is interested in the precession frequency at which depolarization occurs. The time delay of $20-30$ s observed in the beam lifetime measurement compromises this precision measurement of depolarization frequency.

\section{APPENDIX B: DEPOLARIZATION KICKER}

Resonant depolarization of the beam is achieved with a magnetic field which is radial in orientation (perpendicular to both the beam trajectory and main bending field), and oscillating sinusoidally in time. Presented in Fig. 14 is the measured loss rate while scanning the excitation frequency. Exciting the beam at a betatron resonance results in a decreased loss rate (increased lifetime) since the bunch vertical size increases, hence the scattering rate decreases. Crossing a spin resonance the count rate increases since the Møller scattering cross section increases when the polarization is reduced.

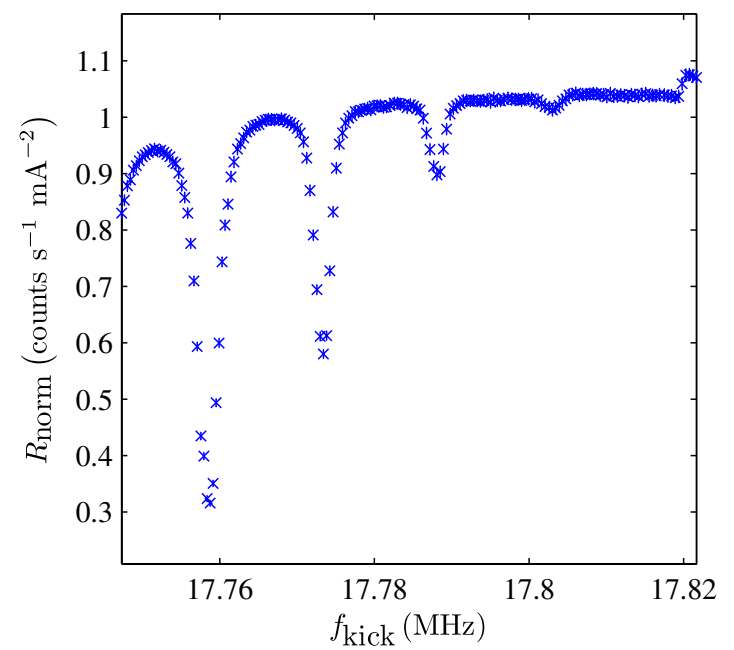

FIG. 14. Measured normalized loss rate resulting from excitation of the beam with a horizontal magnetic field, oscillating sinusoidally in time at the excitation frequency, using the AS storage ring. Large drops in counts correspond to exciting the beam at synchrotron sidebands of the vertical betatron tune. The spin tune was measured as a step increase in normalized loss rate at $17.82 \mathrm{MHz}$. The vertical axis $R_{\text {norm }}$ is normalized according to Eq. (9): the value $R_{\text {norm }}=1$ has no special significance.

For both rings, the excitation frequency was swept at a rate of $10 \mathrm{~Hz} \mathrm{~s}^{-1}$. Depolarization is a resonant effect-it is particularly important to scan slowly [6].

[1] J. Corbett, C. Limborg, Y. Nosochov, J. Safranek, and A. Garren, in Proceedings of European Particle Accelerator Conference 1998 (JACoW, Stockholm, Sweden, 1998), pp. 574-576.

[2] J. W. Boldeman and D. Einfeld, Nucl. Instrum. Methods Phys. Res., Sect. A 521, 306 (2004).

[3] R. Chasman, G. K. Green, and E. M. Rowe, IEEE Trans. Nucl. Sci. 22, 1765 (1975).

[4] C. Steier, J. Byrd, and P. Kuske, in Proceedings of the European Particle Accelerator Conference 2000 (JACoW, Vienna, Austria, 2000), p. MOP5B03.

[5] R. Thornagel, G. Ulm, P. Kuske, T. Mayer, and K. Ott, in Proceedings of the European Particle Accelerator Conference 1994 (JACoW, London, England, 1994), pp. 1719-1721.

[6] P. Kuske, R. Goergen, J. Kuszynski, and P. Schmid, in Proceedings of the International Particle Accelerator Conference 2010 (JACoW, Kyoto, Japan, 2010), p. MOPD083.

[7] S. C. Leemann, M. Böge, M. Dehler, V. Schlott, and A. Streun, in Proceedings of the European Particle Accelerator Conference 2002 (JACoW, Paris, France, 2002), p. TUPRI011.

[8] A.-S. Müller, I. Birkel, E. Huttel, F. Pérez, M. Pont, and R. Rossmanith, in Proceedings of the European Particle Accelerator Conference 2004 (JACoW, Lucerne, Switzerland, 2004), p. THPKF022. 
[9] M. Attal, P. Brunelle, A. Loulergue, A. Nadji, L. Nadolski, and M.-A. Tordeux, Phys. Rev. ST Accel. Beams 16, 054001 (2013).

[10] R. Belbéoch, M. Bergher, J. Le Duff, M. P. Level, P. Mann, C. Nguyen Ngoc, M. Sommer, and H. Zyngier, in Proceedings of the Soviet Conference on ChargedParticle Accelerators, Moscow, Russia, 1968 (Israel Program for Scientific Translations, Jerusalem, Israel, 1972), pp. 130-134.

[11] The Orsay Storage Ring Group, in Proceedings of the 8th International Conference on High-Energy Accelerators (CERN, Geneva, Switzerland, 1971), pp. 127-131.

[12] S. I. Serednyakov, A. N. Skrinsky, G. M. Tumaikin, and Y. M. Shatunov, Zh. Eksp. Teor. Fiz. 71, 2025 (1976) [Sov. Phys. JETP 44, 1063 (1976)].

[13] Y.S. Derbenev, A. M. Kondratenko, S. I. Serednyakov, A. N. Skrinsky, G. M. Tumaikin, and Y.M. Shatunov, Part. Accel. 10, 177 (1980).

[14] J. R. Johnson, R. Prepost, D. E. Wiser, J. J. Murray, R. F. Schwitters, and C. K. Sinclair, Nucl. Instrum. Methods 204, 261 (1983).

[15] L. Arnaudon, B. Dehning, P. Grosse-Wiesmann, R. Jacobsen, M. Jonker, J. Koutchouk, J. Miles, R. Olsen, M. Placidi, R. Schmidt, J. Wenninger, R. Assmann, and A. Blondel, Z. Phys. C 66, 45 (1995).

[16] I. P. S. Martin, M. Apollonio, R. T. Fielder, G. Rehm, and R. Bartolini, in Proceedings of the International Particle Accelerator Conference 2011 (JACoW, San Sebastian, Spain, 2011), p. TUPC159.

[17] J. Zhang, L. Cassinari, M. Labat, A. Nadji, L. S. Nadolski, and D. Pédeau, in Proceedings of the International Particle Accelerator Conference 2011 (Ref. [16]), p. THPC006.

[18] J. Zhang, L. Cassinari, M. Labat, A. Nadji, L. S. Nadolski, and D. Pédeau, Nucl. Instrum. Methods Phys. Res., Sect. A 697, 1 (2013).

[19] G. Vignola, Nucl. Instrum. Methods Phys. Res., Sect. A 246, 12 (1986).

[20] A. Jackson, S. Chattopadhyay, R. Keller, C. Kim, H. Nishimura, F. Selph, and M. Zisman, in Proceedings of European Particle Accelerator Conference 1988 (JACoW, Rome, Italy, 1988), pp. 359-361.

[21] M. Yoon, J. Corbett, M. Cornacchia, J. Tanabe, and A. Terebilo, Nucl. Instrum. Methods Phys. Res., Sect. A 523, 9 (2004).

[22] D. Einfeld, M. Belgroune, G. Benedetti, M. D. Lima, J. Marcos, M. Munoz, and M. Pont, in Proceedings of Particle Accelerator Conference 2007 (JACoW, Albuquerque, NM, 2007), p. TUPMN068.

[23] Y. Chen, D. E. Kim, W. Kang, F. S. Chen, M. Yang, Z. Zhang, B. G. Yin, and J. X. Zhou, Nucl. Instrum. Methods Phys. Res., Sect. A 682, 85 (2012).

[24] S. C. Leemann, A. Andersson, M. Eriksson, L.-J. Lindgren, E. Wallén, J. Bengtsson, and A. Streun, Phys. Rev. ST Accel. Beams 12, 120701 (2009).

[25] S. C. Leemann and A. Streun, Phys. Rev. ST Accel. Beams 14, 030701 (2011).

[26] Y. Nosochkov, K. L. F. Bane, Y. Cai, R. Hettel, and M.-H. Wang, in Proceedings of the International Particle Accelerator Conference 2011 (Ref. [16]), p. THPC075.
[27] M.-H. Wang, Y. Nosochkov, K. Bane, Y. Cai, R. Hettel, and X. Huang, Nucl. Instrum. Methods Phys. Res., Sect. A 649, 30 (2011).

[28] Y. Cai, K. Bane, R. Hettel, Y. Nosochkov, M.-H. Wang, and M. Borland, Phys. Rev. ST Accel. Beams 15, 054002 (2012).

[29] E. D. Courant and H. S. Snyder, Ann. Phys. (N.Y.) 3, 1 (1958).

[30] S. R. Mane, Y. M. Shatunov, and K. Yokoya, Rep. Prog. Phys. 68, 1997 (2005).

[31] A. A. Sokolov and I. M. Ternov, Dokl. Akad. Nauk SSSR 153, 1052 (1963) [Sov. Phys. Dokl. 8, 1203 (1964)].

[32] R. H. Helm, M. J. Lee, P. L. Morton, and M. Sands, in Proceedings of Particle Accelerator Conference 1973 (JACoW, San Francisco, CA, 1973), pp. 900-901.

[33] V. Bargmann, L. Michel, and V.L. Telegdi, Phys. Rev. Lett. 2, 435 (1959).

[34] B. Odom, D. Hanneke, B. D'Urso, and G. Gabrielse, Phys. Rev. Lett. 97, 030801 (2006).

[35] P. J. Mohr, B. N. Taylor, and D. B. Newell, Rev. Mod. Phys. 84, 1527 (2012).

[36] A. W. Chao and M. Tigner, Handbook of Accelerator Physics and Engineering (World Scientific Publishing, Singapore, 1999).

[37] V. N. Baier and V. A. Khoze, At. Energ. 25, 440 (1968) [Sov. J. At. En. 25, 1256 (1968)].

[38] G. W. Ford and C. J. Mullin, Phys. Rev. 108, 477 (1957).

[39] G. W. Ford and C. J. Mullin, Phys. Rev. 110, 1485 (1958).

[40] C. Bernardini, G. F. Corazza, G. Di Giugno, G. Ghigo, J. Haissinski, P. Marin, R. Querzoli, and B. Touschek, Phys. Rev. Lett. 10, 407 (1963).

[41] H. P. Panopoulos, K. P. Wootton, M. J. Boland, and R.P. Rassool, in Proceedings of the International Particle Accelerator Conference 2011 (Ref. [16]), p. TUPC062.

[42] K. P. Wootton, M. J. Boland, R. P. Rassool, Y.-R. E. Tan, W. J. Corbett, M. Donald, X. Huang, R. R. Ortiz, J. A. Safranek, and K. Tian, in Proceedings of the International Particle Accelerator Conference 2012 (JACoW, New Orleans, 2012), p. MOPPR001.

[43] E. D. Courant, M.S. Livingston, and H. S. Snyder, Phys. Rev. 88, 1190 (1952).

[44] H. Wiedemann, Particle Accelerator Physics (SpringerVerlag, Berlin, Germany, 2007), 3rd ed.

[45] X. Huang, J.A. Safranek, and D. Dell Orco, in Proceedings of the International Particle Accelerator Conference 2010 (Ref. [6]), p. THPE048.

[46] A. Terebilo, Accelerator Toolbox for MATLAB (Stanford Linear Accelerator Center, Stanford University, Stanford, CA, 2001).

[47] X.R. Resende, R. Basílio, L. Liu, P. P. Sanchez, and G. Tosin, in Proceedings of the International Particle Accelerator Conference 2012 (Ref. [42]), p. MOPPC053.

[48] S. Machida et al., Nat. Phys. 8, 243 (2012).

[49] G. H. Rees, D. J. Kelliher, S. Machida, C. R. Prior, and S.L. Sheehy, in Proceedings of the International 
Particle Accelerator Conference 2012 (Ref. [42]), p. MOPPD020.

[50] S. L. Sheehy, K. J. Peach, H. Witte, D. J. Kelliher, and S. Machida, Phys. Rev. ST Accel. Beams 13, 040101 (2010).

[51] K. J. Peach et al., Phys. Rev. ST Accel. Beams 16, 030101 (2013).
[52] M. Xiao, in Proceedings of the International Particle Accelerator Conference 2012 (Ref. [42]), p. TUPPR085.

[53] J. G. Wang, Nucl. Instrum. Methods Phys. Res., Sect. A 722, 87 (2013).

[54] V. N. Bă̌er, Usp. Fiz. Nauk 105, 441 (1971) [Sov. Phys. Usp. 14, 695 (1972)]. 